\title{
A Review of Common Local Flaps for Coverage of Skin Defects of the Nose: Clinical Experience in Ten Cases
}

\author{
AHMED ABD EL-SALAM HAFIZ, M.D. and AHMED MAHER SAMI, M.D. \\ The Department of General Surgery, Plastic Surgery Unit, Faculty of Medicine, Cairo University
}

\begin{abstract}
The nose is considered an important structure in the face from both the cosmetic and functional point of view. It is the most projecting part in the face; hence any disfigurement is obvious and cannot be hidden. Skin defects may occur due to many reasons including post traumatic and post ablative following excision of skin cancer. There are various methods for coverage according to the reconstructive ladder. However, due to its importance, local flaps are always preferable to skin grafts as this produces like with like replacement including good padding with well vascularized tissue, pliable coverage, sensate tissue with better contouring, texture and color matching when compared to skin grafting. In this paper, although many flaps are described in literature, but most defects of the nose can be best closed by three common flaps used in this area. These flaps are reviewed including forehead flap, nasolabial flap and bilobed flap with the authors' personal experience in this field. Ten cases were operated upon by the authors in Kasr Al-Aini Hospital between February 2016 to July 2016 and followed-up for the following six months. All these flaps survived with a satisfactory patient outcome in most patients.
\end{abstract}

Key Words: Nasal skin defects - Aesthetic subunits - Full thickness skin graft - Forehead flap - Nasolabial flap-Bilobed flap.

\section{INTRODUCTION}

Nasal skin defects can occur due to many reasons. Common etiology includes post traumatic and post ablative for skin cancer. The most common non melanoma skin cancer is basal cell carcinoma, $80 \%$ occurring in the face among which $25 \%$ involving the nose. Surgical excision with adequate safety margin around 0.5 centimeter to achieve $95 \%$ cure rate is the main line of treatment resulting in a defect that may require coverage if too large and primary closure is not feasible [1,2].

The nose is divided into nine subunits from the aesthetic point of view according to Burget and Menick [3,4]. Defects of adjacent aesthetic subunits require soft tissue replacement of the affected subunit and preservation of the naturally occurring boundaries thus resulting in superior aesthetic results when these subunits are individually reconstructed with the flap interface placed precisely at the naturally occurring subunit boundary [5].

There are various modalities for coverage of nasal skin defects from full thickness skin grafts to local or distant flaps for resurfacing of defects. The results of skin grafts are less than satisfactory for large areas to cover and distant flaps are bulky with a poor color match. It usually takes many weeks for skin grafts to stabilize and match with the recipient site. Contractures may develop in the long-term follow-up [6]. Local skin flaps provide excellent choice for coverage of nasal skin defects with good texture, color match and success rate. It has the additional advantage of well vascularized tissue coverage of the nasal skeleton resisting contracture and infection. The reconstructive option varies according to the defect site, defect size, skin laxity, tissue availability, age of patient and general condition. Flap selection is individualized based on the previous mentioned factors as no single flap can be considered universal for the nasal defect. The common flaps including forehead, nasolabial and bilobed flaps are reviewed [7].

\section{PATIENTS AND METHODS}

This is a retrospective study of ten patients (seven males and three females). Their age was from ten to sixty five with mean age of fifty. These patients had nasal defects of various etiologies (one post traumatic and nine postresection of basal cell carcinoma). The various nasal aesthetic subunits were involved as the following: Three nasal tip with basal cell carcinoma: Two had bilobed flaps and one had a forehead flap, one posttraumatic loss of columella had a superiorly based nasolabial flap, three dorsal and one sidewall basal cell car- 
cinoma: They had forehead flaps and two nasal alae basal cell carcinoma had nasolabial flaps. All these patients were operated upon in Kasr Al-Aini Hospital, Faculty of Medicine, Cairo University from February 2016 to July 2016 and followed-up for six months. All nine cases with basal cell cancer were excised with frozen section technique showing adequate safety margin and no evidence of regional or distant metastasis and coverage was done in the same setting. A patient had iatrogenic loss of the columella due to pressure atrophy by the nasal prongs when he was an infant in the incubator. All cases were done on elective bases.

\section{Surgical technique:}

All cases were done under general anesthesia except one female, sixty year old, cardiac patient with basal cell cancer of the nasal tip. A bilobed flap was done under local anesthesia. Diluted adrenaline was injected around the tumor to achieve hemostasis. The scalpel was changed after tumor resection and a new one was used for flap elevation.

All flaps were done in a peninsula skin design. The forehead flaps were done in a staged manner while in the nasolabial flap: Two cases were done in a staged manner and one case in a single stage. The second stage of the flap was done after three weeks which entails excision of the skin bridge. The bilobed flap was done in a single stage operation. No drains were inserted. Betadine wet gauze was applied around the bridge of skin between the pedicle and inset recipient site to avoid infection of the raw undersurface of the flap. No tubing of the pedicle was done to limit flap congestion. In the forehead flaps, the donor areas closed primary with excessive undermining of the forehead skin. All patients had smooth post-operative recovery.

\section{RESULTS}

All flaps survived completely but one case of forehead flap showed mild distal congestion that improved later on. No recurrence of malignancy, no infections or hematomas were encountered. Two cases of forehead flap and one case of nasolabial flap showed initial bulky appearance and debulking was done after 3-6 months. All patients had satisfactory cosmetic and functional outcomes except two patients: One with persistent bulky forehead flap due to flap lymphedema and another due to forehead scar. Table (1) showing summary of the cases.

Sample of pre-operative and post-operative cases are shown below Figs. (1-5).

Table (1): Showing summary of cases.

\begin{tabular}{|c|c|c|c|c|c|c|c|}
\hline Case & Sex & Age & Etiology & Subunit involved & Type of flap & Complications & Patient satisfaction \\
\hline 1 & Male & 44 & Basal cell cancer & Dorsum & Forehead & None & Satisfied \\
\hline 2 & Male & 10 & Traumatic & Columella & Nasolabial & None & Satisfied \\
\hline 3 & Female & 60 & Basal cell cancer & Tip & Bilobed & None & Satisfied \\
\hline 4 & Female & 47 & Basal cell cancer & Lateral sidewall & Forehead & Forehead scar & Unsatisfied \\
\hline 5 & Male & 65 & Basal cell cancer & Tip & Bilobed & None & Satisfied \\
\hline 6 & Male & 57 & Basal cell cancer & Dorsum & Forehead & None & Satisfied \\
\hline 7 & Male & 53 & Basal cell cancer & Tip and soft tissue triangle & Forehead & Distal Flap congestion & Satisfied \\
\hline 8 & Male & 59 & Basal cell cancer & Dorsum & Forehead & Bulky flap (lymphedema) & Unsatisfied \\
\hline 9 & Male & 41 & Basal cell cancer & Right ala & Nasolabial & None & Satisfied \\
\hline 10 & Female & 63 & Basal cell cancer & Left ala & Nasolabial & None & Satisfied \\
\hline
\end{tabular}

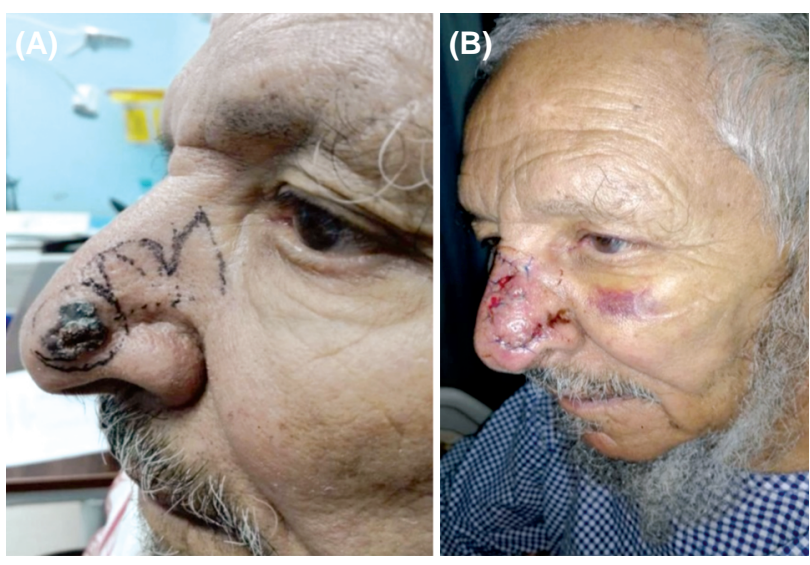

Fig. (1): 65 year old male patient with basal cell cancer of the nasal tip. (A) Pre-operative marking of the bilobed flap. (B) Post-operative picture.

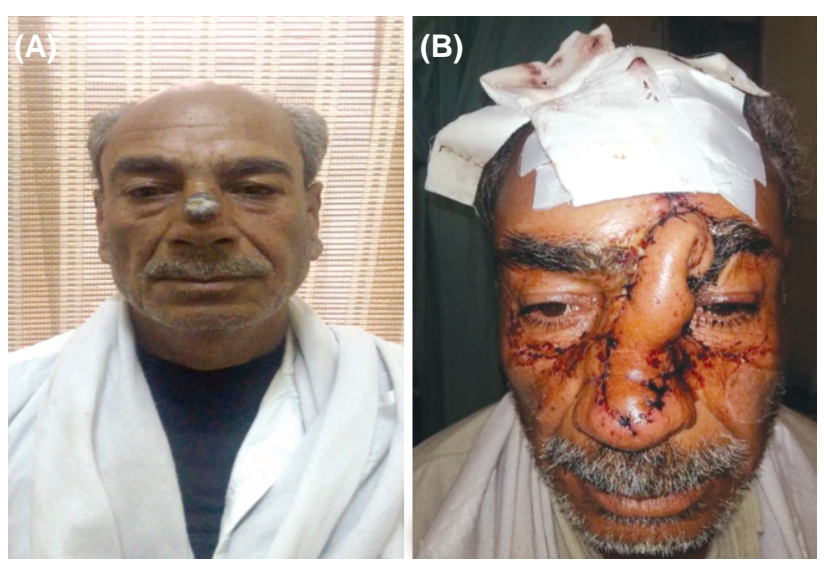

Fig. (2): 57 year old male patient with basal cell cancer of the nasal dorsum (A) Pre-operative picture (B) Postoperative first stage forehead flap. 

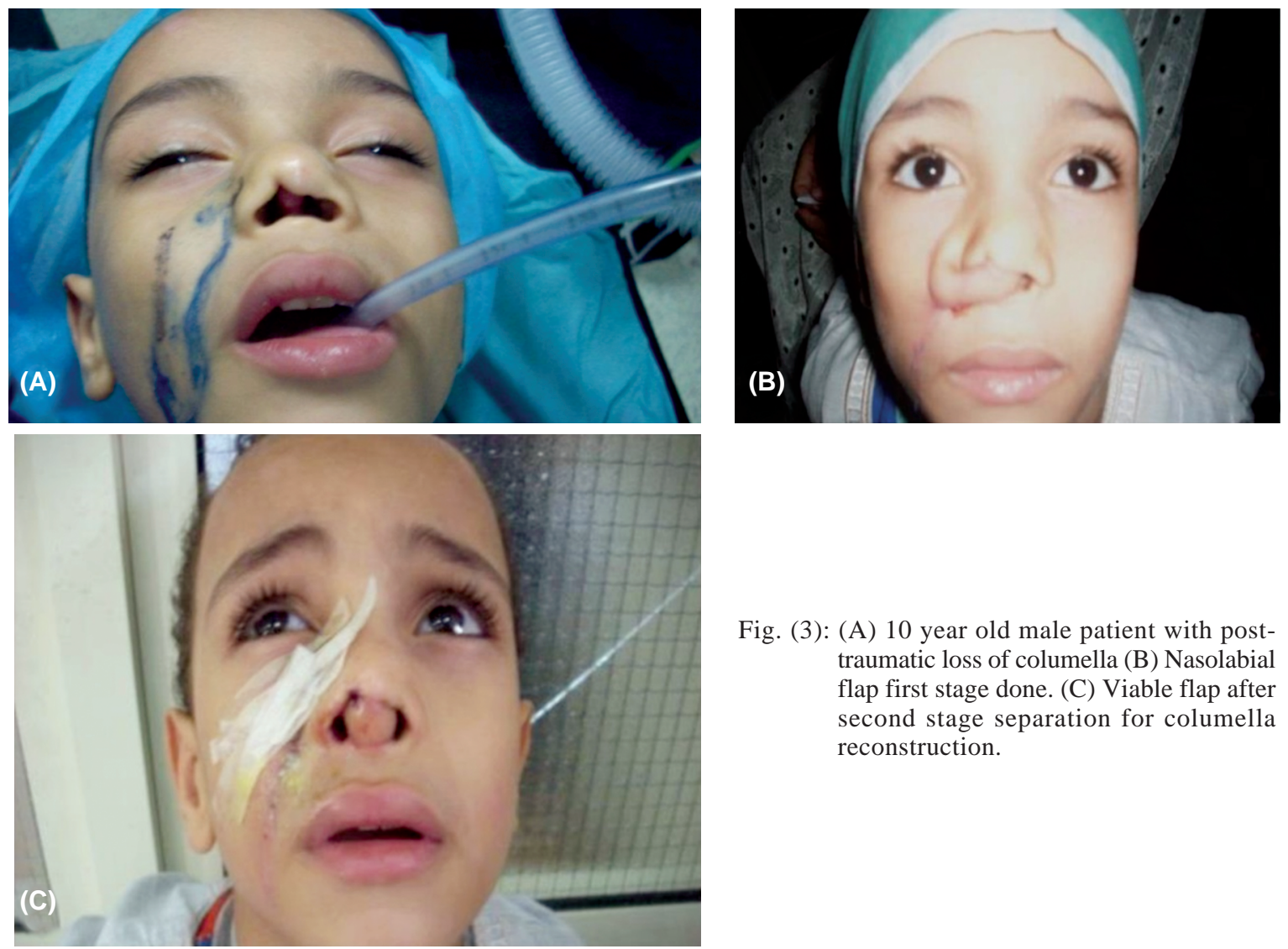

Fig. (3): (A) 10 year old male patient with posttraumatic loss of columella (B) Nasolabial flap first stage done. (C) Viable flap after second stage separation for columella reconstruction.
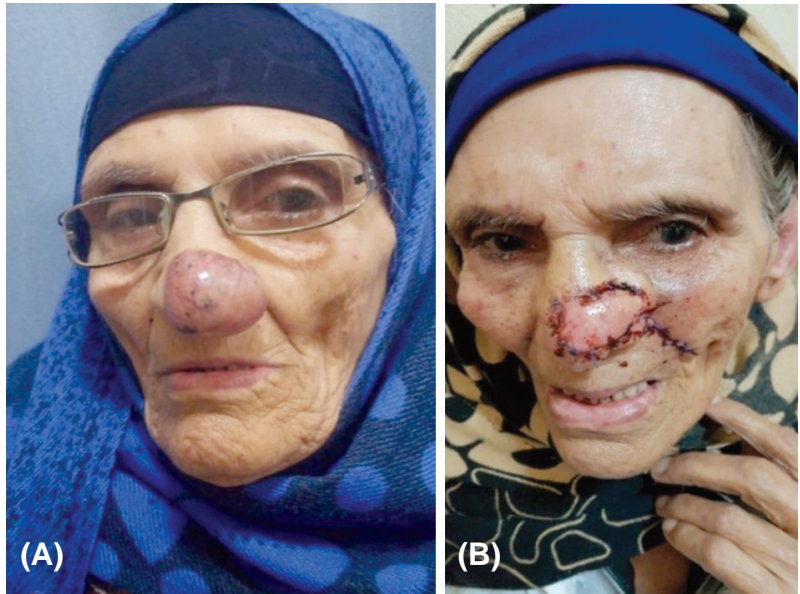

Fig. (4): (A) Pre-operative picture of a 63 year old female patient with rodent ulcer (infundibulocystic type) involving nasal tip and left ala. (B) Post-operative picture of nasolabial flap.

\section{DISCUSSION}

The face is the most important area in the human body from the cosmetic point of view. The nose is the most projecting part in the face, hence the importance from the aesthetic and functional point of view. Skin defects in the nose may occur due

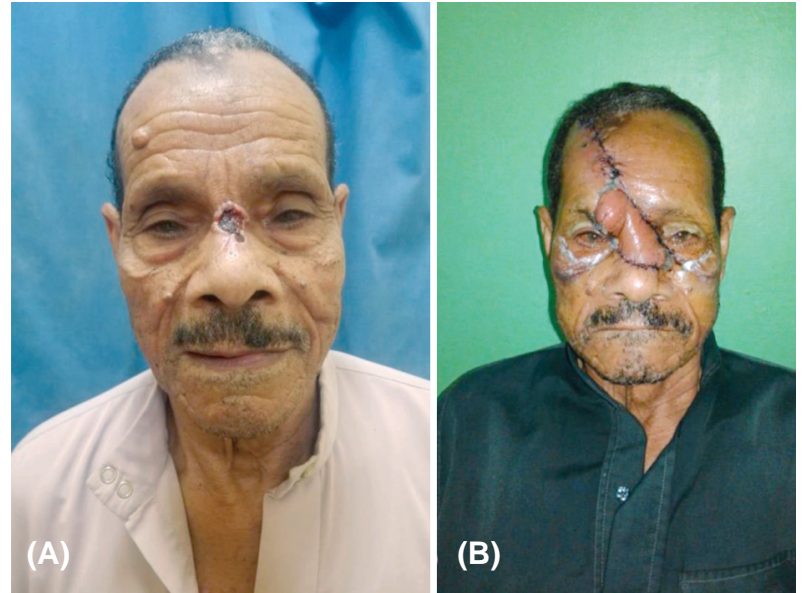

Fig. (5): (A) Pre-operative picture of 59 year old male patient with rodent ulcer of nasal dorsum (B) Post-operative picture of median forehead flap (bulky lymphedema).

to various etiology especially trauma and following malignant resection.

Aesthetic units of the nose should be taken into consideration during skin coverage. Also the structural part of the skin which is loose and highly mobile on the bony part and adherent to the nasal 
cartilages. Healing by secondary intention of convex surfaces like the nasal tip should be avoided since healing may lead to uneven scars and irregularities [8].

Full thickness skin grafting results are less satisfactory for large areas to cover and it usually takes several weeks to stabilize and match with the recipient site. Contracture in the form of hypertrophic scar and keloid may develop. Also graft loss due to infection may occur [6].

Local flaps are the first choice for coverage of nasal skin defects as they provide excellent cosmetic results through the matching of the skin color, texture and structural characteristics. They also have a very rich blood supply with minimal complications and do not require microvascular expertise $[\mathbf{9 , 1 0}]$.

The nasolabial flap based on the perpendicular perforating branches of the facial artery, may be superiorly or inferiorly based. The superiorly based flap is used for coverage of defects of the nasal alae, tip and sidewalls while the inferiorly based flap is used for defects in the nasal floor and columella. The donor site scar is hidden in the nasolabial groove. One advantage of this flap is that it can be used for single stage reconstruction of the lateral alar defect as transposition-advancement flap [11]. The skin in melolabial fold has the nature to contract which is useful to maintain the round bulging of the new ala. However, a blunt alar groove and development of trapdoor deformity are some disadvantages of this flap [12]. In our personal experience, a patient with lost columella, a superiorly based long nasolabial flap reaching down to the mesolabial fold was used. This long flap was used in order to reach the columella subunit from outside, thus avoiding an incision in the superior alar groove and tunneling that may interfere with flap vascularity. In another case with infundibulucystic type of rodent ulcer, a single stage transposition nasolabial flap was done due to absent intervening tissue between the flap and the defect.

The forehead flap is based on the supratrochlear and supraorbital vessels. This flap is a powerful tool in nasal reconstruction and is the method of choice for coverage of large, distal nasal defects. It has evolved from the ancient roots as a broad based flap of excessive bulk and significant donor site morbidity to an elegant shape having a narrow pedicle with adequate length and appropriate thickness done in a single stage manner in the form of a skin island resulting in an aesthetically pleasing cosmetic result. The midline paddle has advantage of a favorable donor site scar [13]. In our personal experience, this flap was done in a staged procedure: Flap elevation, separation and sometimes debulking. It was in the form of a peninsula aiming to improve flap vascularity by avoiding tunneling and maintaining the subdermal venous plexus. However one patient developed persistent bulky lymphedema after flap separation. A single stage median forehead flap should be taken into consideration.

The bilobed flap was first described by Esser in 1918 that used it to cover the nasal tip. Zitelli modified the design by reducing the rotational angle and it became the workhorse flap for $1-1.5 \mathrm{~cm}$ defect of the distal and lateral part of the nose particularly for the nasal tip. This flap has good cosmetic appearance, single stage and easy surgical technique $[\mathbf{1 4 , 1 5}]$. In our personal experience, this flap is easy to elevate but needs good planning and marking. It can be used in distal nasal defects not exceeding two centimeters in diameters.

\section{Conclusion:}

The choice of the flap depends on the nasal subunits involved, size of the defect, availability of the flap, the resulting donor site morbidity and distortion and finally the general condition of the patient. More than one flap could be used in the same patient to cover the involved multiple nasal subunits. Although many flaps are described in the literature for skin coverage of nasal defects, most defects can be best closed by forehead, nasolabial and bilobed flaps. Good cosmetic and functional outcomes can be achieved. Hence, local flaps are considered the workhouse for nasal coverage placing them at a prior step in the reconstructive ladder. In our experience, local flaps are the first choice for coverage of nasal skin defects with similar acceptable results to the universal standard ones.

\section{REFERENCES}

1- Netsher D.T. and Spira M.: Basal cell carcinoma: An overview of tumor biology and treatment. Plast. Reconst. Surg., 113: 70-94, 2004.

2- Wolf D.J. and Zitelli J.A.: Surgical margins for basal cell carcinoma. Arch. Dermatolol., 123: 340-344, 1987.

3- Burget G.C. and Menick F.J.: The subunit principle in nasal reconstruction. Plast. Reconstr. Surg., 76: 239-247, 1985.

4- Burget G.C.: Aesthetic restoration of the nose. Clin. Plast. Surg., 12: 463-480, 1985.

5- Zelken J.A., et al.: Nasolabial and forehead flap reconstruction of contiguous alar-upper lip defects. Journal of Plastic, Reconstructive and Aesthetic Surgery, 70: 330$335,2017$. 
6- Gloster H.M. Jr.: The use of full thickness skin grafts to repair non perforating nasal defects. Am. Acad. Dermatol., 42: 1041-1050, 2000.

7- Zitelli J.A. and Fazio M.J.: Reconstruction of the nose with local flaps. J. Dermatol. Surg. Oncol., 17: 184-189, 1991.

8- Mott K.J., Clark D.P. and Stelljes L.S.: Regional variation in wound contraction of mohs surgery defects allowed to heal by second intention. Dermatol. Surg., 29: 712-722, 2003.

9- Heniford B.W., Bailin P.L. and Marisco Jr. R.E.: Field guide to local flaps. Dermatol. Clin., 16: 65-74, 1998.

10- Schliephake H., Neukam F.W., Schmelzeisen R. and Reiche C.: Reconstruction of facial soft tissues after resection of skin tumors. J. Craniomaxillofacial. Surg., 22: 342-348, 1994.

11- Zitelli J.A.: The nasolabial flap as a single stage procedure. Arch. Dermatol., 126: 1445-1448, 1990.

12- Rieger R.A.: A local flap for repair of nasal tip. Plast. Reconst. Surg., 40: 147-149, 1967.

13- Rasamny J.K.J. and Park Stephen S.: Refinements of the forehead flap in nasal reconstruction: Operative techniques in otolaryngology, 22: 65-71, 2011.

14- Cook J.L.: Reconstructive utility of the bilobed flap: Lessons from flap successes and failures. Dermatol. Surg., 31: 1024-1033, 2005.

15- Cook J.L.: A review of the bilobed flaps design with particular emphasis on the minimization of alar displacement. Dermatol. Surg., 26: 354-362, 2000. 